\title{
METHODOLOGY FOR PREDICTING CHEMICAL EQUILIBRIA SHIFT: CHANGING SYSTEM COMPOSITION AT CONSTANT TEMPERATURE
}

\author{
SOLAZ-PORTOLÉS, Joan Josep ${ }^{1 *}$; CAURIN, Carlos² \\ 1,2 Universitat de València, Facultat de Magisteri, Departament de Didàctica de les Ciències Experimentals $\mathrm{i}$ \\ Socials, Avgda Tarongers, 4, 46022 València - País Valencià, España. \\ (fone: +34 963864483 ) \\ *e-mail: Joan.Solaz@uv.es
}

Received 20 May 2012; received in revised form 13 July 2012; accepted 20 July 2012

\section{RESUMEN}

El tratamiento de las perturbaciones de un sistema en equilibrio y el sentido del desplazamiento hacia un nuevo equilibrio es una cuestión muy importante en los cursos de Química General y Fisicoquímica. Resulta de particular interés la preparación de los estudiantes para prever los efectos del cambio en las condiciones del sistema sobre la posición del equilibrio químico. En el contexto educativo, el principio de Le Chatelier es todavía utilizado como un principio infalible, sin mostrar que sus limitaciones pueden conducir a destacados errores conceptuales. En este trabajo se presenta una metodología de enseñanza que permite evitar el principio de Le Chatelier como regla cualitativa. Esta metodología se basa en el grado de avance de la reacción, la afinidad de la reacción y los potenciales termodinámicos. Nuestra propuesta, que conduce a la expresión matemática de la variación del grado de avance de la reacción con el cambio infinitesimal en el número de moles, permite efectuar predicciones exactas sobre la evolución de sistemas en equilibrio químico que han sido perturbados. En concreto, se analizan los efectos de la adición de reactivos o productos sobre el equilibrio químico (a temperatura constante).

Palabras-clave: Propuesta didáctica, análisis termodinámico, evolución de reacciones perturbadas, adición de componentes activos.

\begin{abstract}
The subject of perturbations of an equilibrium system and the direction in which it shifts as it moves towards a new equilibrium is an important aspect of general and physical chemistry courses. Of particular importance is students' ability to predict the effects of changing conditions on the position of chemical equilibrium. On a teaching context, the Le Chatelier's principle is still used as an infallible principle without showing its limitations which can lead to very important misconceptions. In this paper it is presented a teaching methodology to avoid the Le Chatelier's qualitative rule. This methodology is based on extent of reaction, affinity of reaction, and thermodynamic potentials. Focusing on the effects of adding more reactants or products on chemical equilibrium (at constant temperature), our proposal gives the mathematical expression of the variation of the extent of reaction with the infinitesimal variation in the number of moles, and it allows to make exact predictions about the evolution of a perturbed chemical equilibrium system.
\end{abstract}

Keywords: Teaching proposal, thermodynamic analysis, evolution of perturbed reactions, adding active component. 


\section{INTRODUCCIÓN}

La enseñanza y aprendizaje del equilibrio químico resulta de especial complejidad en el currículum de Química (Tyson et al., 1999). Por otro lado, el análisis del desplazamiento del equilibrio químico por la modificación de alguna de las variables que lo definen, se lleva a cabo mayoritariamente en los libros de texto de Química General sobre la base del principio de Le Chatelier como regla cualitativa (Quílez et al., 1993). No obstante, esta formulación cualitativa del principio adolece de graves deficiencias y peca de vaguedad porque no se concretan las condiciones de validez (Solaz-Portolés y Quílez, 1995), lo que puede conducir a una amplia gama de errores conceptuales tanto en estudiantes como en profesores (Quílez y Solaz-Portolés, 1995; Cheung et al., 2007).

Los trabajos de Corti y Franses (2003) y Canagaratna (2003) han intentado, haciendo uso de razonamiento termodinámicos, soslayar la formulación cualitativa del principio de Le Chatelier y acercar el principio hacia formulaciones cuantitativas. Martínez (2007) ha deducido una regla cuantitativa, que se puede aplicar en variables extensivas e intensivas, que permite sustituir com éxito el principio de Le Chatelier.

En general, son muchos los errores conceptuales que cometen los estudiantes preuniversitarios y universitarios en las cuestiones relacionadas con el equilibrio químico (Kousathana y Tsaparlis, 2002; Pereira, 1990; Quílez y Sanjosé, 1995; Quílez, 1998). Además, de acuerdo con Pedrosa y Dias (2000), muchos de estos errores pueden ser encontrados en los libros de texto. Así, Solaz-Portolés (2007) en un análisis de libros de texto de Química españoles pone en evidencia varias deficiencias en el tema de equilibrio químico. También se ha señalado la inadecuada introducción en los textos, tanto desde el punto de vista didáctico como epistemológico, de las constantes de equilibrio químico (Solaz-Portolés, 2010). Por tanto, no resulta muy aventurado afirmar que las dificultades en el equilibrio químico pueden tener su origen en la metodología inadecuada con que se suele presentar en las aulas y en los libros de texto.

Con todo lo dicho hasta aquí, se pone en evidencia la necesidad de una metodología que permita analizar los sistemas en equilibrio y sus perturbaciones de manera cuantitativa y rigurosa. El objetivo de este trabajo es mostrar que tomando como base conceptual los potenciales termodinámicos, la afinidad química y el grado de avance de una reacción podemos prever el sentido de la evolución de los sistemas en equilibrio químico que han sido perturbados, esto es, cómo se desplazarán hasta alcanzar un nuevo equilibrio. La bondad de esta metodología en sistemas cerrados en equilibrio químico ya ha sido puesta de manifiesto en otro artículo (SolazPortolés, 2011). Dado que la mayoría de los libros de texto de Química y Fisicoquímica no incluyen una discusión sobre el caso de que la adición de un reactivo, a presión y temperatura constantes, puede desplazar el equilibrio hacia la formación de más reactivos (Lacy, 2005), en este trabajo aplicaremos dicha metodología a sistemas abiertos, es decir, aquellos que alteran su composición por la adición o eliminación de una especie activa del equilibrio químico.

\section{GRADO DE AVANCE, AFINIDAD QUÍMICA Y POTENCIALES TERMODINÁMICOS}

Se define el grado de avance de una reacción química como el cociente entre el cambio total de la cantidad de sustancia de una especie activa $i$ y su coeficiente estequiométrico $v_{i}$

$$
\xi=\frac{n_{i}-n_{i o}}{v_{i}}
$$

Se trata de una magnitud que tiene siempre un valor positivo, sus dimensiones son de cantidad de sustancia y su unidad es el mol. La diferenciación de esta última expresión, donde $n_{i, 0}$ y $v_{i}$ son constantes, conduce a

$$
d \xi=\frac{d n_{i}}{v_{i}}
$$

Puede verse que si una reacción química procede de reactivos a productos $d \xi>0$, ya que numerador y denominador son negativos.

La afinidad química o de reacción representa la variación del potencial termodinámico que gobierna el comportamiento 
del sistema con el grado de avance de la reacción cambiada de signo, manteniendo constantes las restantes variables naturales. En concreto, si la temperatura y la presión son constantes tenemos

$$
\mathrm{A}=-\Delta_{r} G=-\left(\frac{\partial G}{\partial \xi}\right)_{T, P}
$$

donde $\mathrm{G}$ es el potencial termodinámico energía libre de Gibbs. Si la temperatura y el volumen son constantes

$$
\mathrm{A}=-\left(\frac{\partial F}{\partial \xi}\right)_{T, V}
$$

donde $\mathrm{F}$ es el potencial termodinámico energía libre de Helmholtz.

Así, cuando el sistema se define mediante las variables independientes $\mathrm{T}, \mathrm{P}$ y $\xi$, la diferencial total de la afinidad química $(d A)$ se podrá escribir

$$
\begin{aligned}
& d A= \\
& \left(\frac{\partial A}{\partial T}\right)_{P, \xi} d T+\left(\frac{\partial A}{\partial P}\right)_{T, \xi} d P+\left(\frac{\partial A}{\partial \xi}\right)_{T, P} d \xi
\end{aligned}
$$

en tanto que si las variables naturales del sistema son $\mathrm{T}, \mathrm{V}$ y $\xi$, la diferencial total será posible expresarla

$d A=$

$$
\left(\frac{\partial A}{\partial T}\right)_{V, \xi} d T+\left(\frac{\partial A}{\partial V}\right)_{T, \xi} d V+\left(\frac{\partial A}{\partial \xi}\right)_{T, V} d \xi
$$

Por otro lado, teniendo presentes las diferenciales de los potenciales termodinámicos energía libre de Gibbs y de Helmholtz

$$
\begin{aligned}
& d G=-S d T+V d P-\mathrm{A} d \xi \\
& d F=-S d T-P d V-\mathrm{A} d \xi
\end{aligned}
$$

y la igualdad de las derivadas parciales segundas cruzadas, se coligen las siguientes expresiones

$$
\begin{aligned}
& \left(\frac{\partial A}{\partial T}\right)_{P, \xi}=-\left[\frac{\partial}{\partial T}\left(\frac{\partial G}{\partial \xi}\right)_{T, P}\right]_{P, \xi}= \\
& -\left[\frac{\partial}{\partial \xi}\left(\frac{\partial G}{\partial T}\right)_{P, \xi}\right]_{T, P}=\left(\frac{\partial S}{\partial \xi}\right)_{T, P}=\Delta_{r} S
\end{aligned}
$$

siendo $\Delta_{r} S$ la variación de entropía de la reacción, y

$$
\begin{aligned}
& \left(\frac{\partial A}{\partial P}\right)_{T, \xi}=-\left[\frac{\partial}{\partial P}\left(\frac{\partial G}{\partial \xi}\right)_{T, P}\right]_{T, \xi}= \\
& -\left[\frac{\partial}{\partial \xi}\left(\frac{\partial G}{\partial P}\right)_{T, \xi}=-\left(\frac{\partial V}{\partial \xi}\right]_{T, P}=-\Delta_{r} V\right.
\end{aligned}
$$

siendo $\Delta_{r} V$ la variación del volumen de la reacción

ecuaciones

Y también se deducen estas otras

$$
\begin{aligned}
& \left(\frac{\partial A}{\partial T}\right)_{V, \xi}=-\left[\frac{\partial}{\partial T}\left(\frac{\partial F}{\partial \xi}\right)_{T, V}\right]_{V, \xi}= \\
& -\left[\frac{\partial}{\partial \xi}\left(\frac{\partial F}{\partial T}\right)_{V, \xi}\right]_{T, V}=\left(\frac{\partial S}{\partial \xi}\right)_{T, V}
\end{aligned}
$$

$$
\begin{aligned}
& \left(\frac{\partial A}{\partial V}\right)_{T, \xi}=-\left[\frac{\partial}{\partial V}\left(\frac{\partial F}{\partial \xi}\right)_{T, V}\right]_{T, \xi}= \\
& -\left[\frac{\partial}{\partial \xi}\left(\frac{\partial F}{\partial V}\right)_{T, \xi}=\left(\frac{\partial P}{\partial \xi}\right)_{T, V}\right.
\end{aligned}
$$

Con lo cual, efectuando substituciones pertinentes en las expresiones diferenciales (Ec.5) y (Ec.6), son susceptibles de ser formuladas del modo siguiente

$$
d A=\Delta_{r} S \cdot d T-\Delta_{r} V \cdot d P+\left(\frac{\partial A}{\partial \xi}\right)_{T, P} d \xi
$$

$$
d A=
$$$$
\left(\frac{\partial S}{\partial \xi}\right)_{T, V} d T+\left(\frac{\partial P}{\partial \xi}\right)_{T, V} d V+\left(\frac{\partial A}{\partial \xi}\right)_{T, V} d \xi
$$ 
Ecuaciones con las que trabajaremos en el análisis de los efectos de perturbaciones del equilibrio de una mezcla de gases ideales.

\section{EQUILIBRIO Y EVOLUCIÓN DEL EQUILIBRIO}

Teniendo presente que las diferenciales de los potenciales termodinámicos (Ec. 7) y (Ec. 8) en los procesos irreversibles, efectuados manteniendo constantes sus respectivas parejas de variables naturales $(T, V)$ y $(T, P)$, deben ser negativas, el sentido de la evolución de una reacción química, determinado por el signo de $d \xi$, viene dado por $A$. Un sistema cuya afinidad química es positiva evolucionará en el sentido correspondiente a un aumento en el grado de avance de la reacción $(d \xi>0)$, es decir, de reactivos a productos. Por el contrario, si $A<0$ entonces $d \xi<0$, y el sentido de la evolución será de productos a reactivos.

Cuando un sistema deja de evolucionar macroscópicamente, esto es, ha alcanzado el equilibrio, los potenciales termodinámicos se hacen mínimos y sus diferenciales son iguales a cero. Esto implica que la afinidad química se hace cero. En consecuencia, la condición de equilibrio de un sistema asiento de una reacción química puede escribirse como $A=0$.

Por otro lado, si un sistema ha sido perturbado y se estudia el tránsito entre el estado inicial de equilibrio (estado 1) y el estado de nuevo equilibrio (estado 2), alcanzado tras la perturbación, es obvio que en dicho tránsito no varía la afinidad química $d A=0$. Por ello, en el estudio de los efectos producidos por una perturbación sobre un sistema en químico equilibrio se puede partir de la expresión diferencial de la afinidad e igualarla a cero, y de ahí extraer oportunas conclusiones a través de la variación del grado de avance con la variable que ha sido modificada en la perturbación. Lo veremos a continuación.

\section{MODIFICACIÓN DE LA COMPOSICIÓN A TEMPERATURA CONSTANTE EN UN SISTEMA DE GASES IDEALES EN EQUILIBRIO QUÍMICO.} equilibrio $a A(g)+b B(g)=r R(g)+s S(g)$

para el que podemos escribir (Atkins, 1990)

$A=-R T\left[\ln \frac{n_{R}^{r} \cdot n_{S}^{s}}{n_{A}^{a} \cdot n_{B}^{b}}\left(\frac{P}{P^{0} n}\right)^{\Delta v}-\ln K^{0}\right]$

o también

$$
A=-R T\left[\ln \frac{n_{R}^{r} \cdot n_{S}^{s}}{n_{A}^{a} \cdot n_{B}^{b}}\left(\frac{R \cdot T}{P^{0} \cdot V}\right)^{\Delta v}-\ln K^{0}\right]
$$

donde $P^{\circ}$ es la presión estándar ( 1 bar), $K^{\circ}$ la constante de equilibrio termodinámica y el primer logaritmo corresponde al denominado cociente de reacción, $Q$. La utilización de una u otra expresión estará en función de las ligaduras impuestas a nuestro sistema en la perturbación.

En un sistema abierto se ha de tener presente que la variación de la cantidad de sustancia de cualquier componente activo del equilibrio $\left(d n_{i}\right)$ puede ser atribuido a dos causas: la primera, a la transferencia de materia desde o hacia los alrededores del sistema (da); y la segunda, a la variación en el grado de avance de la reacción $(d \xi)$. Así, por ejemplo, del componente $R(g)$ de nuestra mezcla de gases (Ec.15) tendríamos que la variación de su cantidad de sustancia se expresaría como $d n_{R}=$ $d a+r d \xi$ (Prigogine y Defay, 1954). De este modo, la variación de la afinidad con la composición en los sistemas abiertos vendrá reflejada en dos términos, uno que contendrá la transferencia de materia con el medio externo y otro que recogerá la variación del grado de avance de la reacción.

\section{Adición de una sustancia activa a $T$ y $P$ constantes}

Cuando nuestro sistema en equilibrio es perturbado mediante la adición de una cantidad de sustancia infinitesimal da del componente $R(g)$, a $\mathrm{T}$ y $\mathrm{P}$ constantes, la diferencial de la afinidad será

$d A=\left(\frac{\partial A}{\partial n_{R}}\right)_{T, P, n_{i \neq R}} d n_{R}$ 
que, como $d n_{R}=d a+r d \xi$, se transforma en

$d A=\left(\frac{\partial A}{\partial n_{R}}\right)_{T, P, n_{i \neq R}} d a+\left(\frac{\partial A}{\partial \xi}\right)_{T, P} d \xi$

ya que

$\left(\frac{\partial A}{\partial n_{R}}\right)_{T, P} r \cdot d \xi=\left(\frac{\partial A}{\partial \xi}\right)_{T, P} d \xi$

Por otra parte, de la ecuación 16 obtenemos

$$
\begin{aligned}
& \left(\frac{\partial A}{\partial n_{R}}\right)_{T, P, n_{i \neq R}}=-R T\left(\frac{r}{n_{R}}-\frac{\Delta v}{n}\right)= \\
& -R T\left(\frac{r}{n_{R}}-\frac{y_{R} \cdot \Delta v}{n_{R}}\right)
\end{aligned}
$$

Introduciendo esta derivada parcial en la diferencial (Ec.19) e igualándola a cero, como lícitamente corresponde a un tránsito entre dos estados de equilibrio, nos queda

$-R T\left(\frac{r}{n_{R}}-\frac{y_{R} \cdot \Delta v}{n_{R}}\right) d a+\left(\frac{\partial A}{\partial \xi}\right)_{\mathrm{T}, \mathrm{P}} d \xi=0$ (Ec.22)

de la cual dividiendo por da y reordenando términos se deduce que

$\frac{d \xi}{d a}=\frac{R T}{n_{R} A^{\prime}}\left(r-y_{R} \cdot \Delta v\right)$

donde

$$
A^{\prime}=-\left(\frac{\partial\left(\Delta_{r} G\right)}{\partial \xi}\right)_{T, P}=-\left(\frac{\partial^{2} G}{\partial \xi^{2}}\right)_{T, P}
$$

que, si el equilibrio es considerado estable, es negativa, ya que la derivada segunda de la energía libre de Gibbs es positiva (condición de mínimo del potencial termodinámico energía libre de Gibbs).
Como puede verse, el sentido del desplazamiento del equilibrio tras la adición de $\mathrm{R}(\mathrm{g})$ depende del valor de su fracción molar en el equilibrio. La adición de $\mathrm{R}(\mathrm{g})(\mathrm{da}>0)$ comporta desplazamiento hacia la formación de reactivos $(d \xi<0)$ sólo si $y_{R}<r / \Delta v$. Veamos un ejemplo.

En el equilibrio representado por la ecuación

$\mathrm{CH}_{3} \mathrm{OH}(g)=\mathrm{CO}(g)+2 \mathrm{H}_{2}(g)$

donde $\Delta v=2$, tendremos que si la fracción molar de $\mathrm{CO}(\mathrm{g})$ es mayor que $1 / 2$, la adición de una pequeña cantidad de $\mathrm{CO}(\mathrm{g})$, a $\mathrm{T}$ y $\mathrm{P}$ constantes, provocará la descomposición de más $\mathrm{CH}_{3} \mathrm{OH}(\mathrm{g})$.

\section{Adición de una substancia activa a $T$ y $V$ constantes.}

Como en el caso anterior, la adición de una cantidad de sustancia infinitesimal da del componente $\mathrm{R}(\mathrm{g})$ a $\mathrm{T}$ y $\mathrm{V}$ constantes a nuestro equilibrio de gases, supone una variación elemental de la cantidad de $\mathrm{R}(\mathrm{g})$ recogida en la expresión $d n_{R}=d a+r d \xi$. Operando de manera análoga a la adición a $\mathrm{T}$ y $\mathrm{P}$ constantes, se llega a una variación infinitesimal de la afinidad que puede ser formulada de la siguiente forma

$d A=\left(\frac{\partial A}{\partial n_{R}}\right)_{T, V, n_{i \neq R}} d a+\left(\frac{\partial A}{\partial \xi}\right)_{T, V} d \xi$

Además, de (Ec.17) se obtiene

$\left(\frac{\partial A}{\partial n_{R}}\right)_{T, V, n_{i \neq R}}=-\frac{R T r}{n_{R}}$

que reemplazándola en la diferencial (Ec.26), e igualando esta última a cero por tratarse de un proceso entre dos estados de equilibrio, nos permite concluir que

$\frac{d \xi}{d a}=\frac{R T r}{n_{R} A^{\prime}}$

donde

$A^{\prime}=\left(\frac{\partial A}{\partial \xi}\right)_{T, V}=-\left(\frac{\partial^{2} F}{\partial \xi^{2}}\right)$ 
que, si el equilibrio es considerado estable, es negativa, puesto que la derivada segunda de la energía libre de Helmholtz es positiva (condición de mínimo del potencial termodinámico energía libre de Helmholtz).

La ecuación (14.25) nos indica que la adición de $\mathrm{R}(\mathrm{g})$ ( da $>0$ ) en condiciones de $\mathrm{T}$ y $\mathrm{V}$ constantes siempre desplaza el equilibrio hacia la formación de reactivos $(d \xi<0)$. Veamos un ejemplo

La descomposición térmica del carbamato de amonio puede escribirse como

$\mathrm{NH}_{2} \mathrm{CO}_{2} \mathrm{NH}_{4}(\mathrm{~s})=\mathrm{CO}_{2}(g)+2 \mathrm{NH}_{3}(g)$

para la cual $\Delta v=3$. Según hemos visto anteriormente, si una vez establecido el equilibrio la fracción molar de dióxido de carbono es mayor $1 / 3$, y se añade dióxido de carbono a $T$ y $P$ constantes, el equilibrio evolucionará formando más dióxido de carbono y amoníaco. Si adicionamos amoníaco en las mismas condiciones, este mismo efecto se conseguirá para fracciones molares de dicho gas ep el equilibrio superiores a 2/3. Sin embargo, la adición de cualquiera de los gases a $\mathrm{T}$ 叉 V constantes lleva consigo la formación de ma्ayyor cantidad de carbamato de amonio, sea cual sea la fracción molar del gas en el equilibrio.

\section{CONCLUSIONES Y DISCUSIÓN}

La metodología propuesta nos ha conducido a la posibilidad de llevar a cabo un análisis que permite abordar con precisión y rigor el estudio de sistemas en equilibrio químico tras la perturbación de los mismos (un desarrollo más extenso de este planteamiento junto con multitud de ejemplos prácticos puede encontrarse en SolazPortolés (2002)). Este análisis permite evitar el uso del principio de Le Chatelier como regla meramente cualitativa, que tantos problemas puede generar. No obstante, el tratamiento propuesto presenta cierto grado de complejidad frente a la metafísica simplicidad del enunciado del principio de Le Chatelier que usualmente se presenta en los libros de texto.
Los dos casos analizados en este artículo corresponden a perturbaciones infinitesimales isotérmicas de un componente activo del sistema en equilibrio químico. Sin embargo, los casos más frecuentes que se suelen presentar en el aula de Química, en forma de problemas, son los de perturbaciones finitas. En estos casos, se puede predecir fácilmente el sentido de la evolución del sistema a partir del valor de $A$. Dicho valor puede ser determinado mediante (Ec.16) y (Ec.17) . Esto es tanto como decir que si:

$Q$ (cociente de reacción) $=K^{\circ}$ (constante de equilibrio termodinámica), entonces $A=0$ y el sistema se encuentra en equilibrio $(d \xi=0)$

$Q>K^{\circ}$, entonces $A<0$ y el sistema se desplaza hacia la formación de reactivos $(d \xi<0)$.

$Q<K^{\circ}$, entonces $A>0$ y el sistema se desplaza hacia la formación de productos $(d \xi>0)$.

\section{REFERENCIAS:}

1. Atkins, P.W.; Physical Chemistry, 3rd ed., Oxford University Press: Sussex,1990.

2. Canagaratna, S.G.; J. Chem. Ed., 2003, 80, 1211.

3. Cheung, D.; Ma, H.J.; Yang, J.; Int. J. Sci. Math. Educ., 2009, 7, 1111.

4. Corti, D.S.; Franses, E.I.; Chem. Eng. Ed., 2003, 37, 290.

5. Kousathana, M; Tsaparlis, G.; Chem. Educ. Res. Pract., 2002, 3, 5.

6. Lacy, J.E.; J. Chem. Ed., 2005, 82, 1192.

7. Martínez, E.; J. Chem. Ed., 2007, 84, 516.

8. Pedrosa, M.A.; Dias, M.H.; Chem. Educ. Res. Pract. 2000, 1, 227.

9. Pereira, M.P.B.A.; Equilíbrio químico: dificultades de aprendizagem e sugestões didácticas, 2nd ed., Sociedade Portuguesa de Química: Lisboa, 1990. 
10. Prigogine, I.; Defay, R.; Chemical Thermodynamics, Longman Green: London,1954.

16. Solaz-Portolés, J.J.; Rev. Chil. Educ. Cien., 2007, 6, 13.

11. Quílez, J.; Educ. Quím., 1998, 9, 267.

17. Solaz-Portolés, J.J.; Quim. Bras., 2010, 4, 145.

12. Quílez, J.; Sanjosé, V.; Ens. Cien., 1995, 13, 72.

18. Solaz-Portolés, J.J.; Quim. Nova, 2011, 34, 710.

13. Quílez, J.; Solaz-Portolés, J.J.; Castelló $4 M$.; Sanjosé, V.; Ens. Cien., 1993, 11, $282 . \quad 5$

14. Quílez, J.; Solaz-Portolés, J.J; J. Res. Sci.Teach., 1995, 33, 939.

19. Solaz-Portolés, J.J.; Quílez, J.; Rev. Mex. Fís., 1995, 41, 128.

15. Solaz-Portolés, J.J.; Termodinámica y 20. Tyson, L.; Treagust, D.F.; Bucat, R.B.; J. Chem. Ed., 1999, 76, 554. equilibrio químico, UNED: Valencia, 2002. 